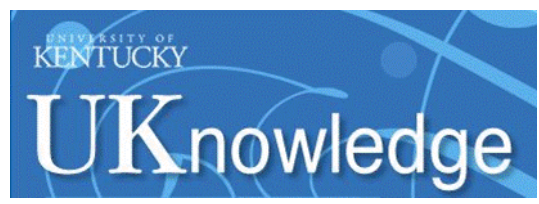

University of Kentucky

UKnowledge

$9-9-2016$

\title{
Skyrmions and Hall Transport
}

\author{
Bom Soo Kim \\ University of Kentucky, bom.soo.kim@uky.edu \\ Alfred D. Shapere \\ University of Kentucky, alfred.shapere@uky.edu
}

Follow this and additional works at: https://uknowledge.uky.edu/physastron_facpub

Part of the Condensed Matter Physics Commons

Right click to open a feedback form in a new tab to let us know how this document benefits you.

\section{Repository Citation}

Kim, Bom Soo and Shapere, Alfred D., "Skyrmions and Hall Transport" (2016). Physics and Astronomy Faculty Publications. 437.

https://uknowledge.uky.edu/physastron_facpub/437

This Article is brought to you for free and open access by the Physics and Astronomy at UKnowledge. It has been accepted for inclusion in Physics and Astronomy Faculty Publications by an authorized administrator of UKnowledge. For more information, please contact UKnowledge@lsv.uky.edu. 


\section{Skyrmions and Hall Transport}

Digital Object Identifier (DOI)

https://doi.org/10.1103/PhysRevLett.117.116805

Notes/Citation Information

Published in Physical Review Letters, v. 117, issue 11, 116805, p. 1-6.

(C) 2016 American Physical Society

The copyright holder has granted permission for posting the article here. 


\title{
Skyrmions and Hall Transport
}

\author{
Bom Soo Kim and Alfred D. Shapere \\ Department of Physics and Astronomy, University of Kentucky, Lexington, Kentucky 40506, USA \\ (Received 15 July 2015; revised manuscript received 17 August 2016; published 9 September 2016)
}

\begin{abstract}
We derive a generalized set of Ward identities that captures the effects of topological charge on Hall transport. The Ward identities follow from the $(2+1)$-dimensional momentum algebra, which includes a central extension proportional to the topological charge density. In the presence of topological objects like Skyrmions, we observe that the central term leads to a direct relation between the thermal Hall conductivity and the topological charge density. We extend this relation to incorporate the effects of a magnetic field and an electric current. The topological charge density produces a distinct signature in the electric Hall conductivity, which is identified in existing experimental data and yields further novel predictions. For insulating materials with translation invariance, the Hall viscosity can be directly determined from the Skyrmion density and the thermal Hall conductivity to be measured as a function of momentum.
\end{abstract}

DOI: 10.1103/PhysRevLett.117.116805

Introduction.-Ward identities in quantum field theories [1] are relations among correlation functions that follow solely from conservation equations and are thus independent of the properties of the Hamiltonian, other than its symmetries. Among their many applications in quantum field theory and many body physics (see, for example, Refs. [2-4]), Ward identities can be used to derive nontrivial relations among various measurable quantities such as conductivities and viscosities. Recently, Ward identities have been applied to $(2+1)$-dimensional systems with broken parity to show that the Hall viscosity is equal to one-half of the angular momentum in the presence of a gap, along with other relations involving transport coefficients [5-11]. In this Letter, we obtain a more general and powerful set of Ward identities for such systems by incorporating topological charges [12] that are not captured by conservation equations. These identities lead to useful relations between transport properties and the density of topological objects such as Skyrmions, with corresponding experimental signatures.

Skyrmions [13-15] in magnetic materials are stable, particlelike spin textures that are protected by topological quantum numbers. They have been studied theoretically [16-22] and have been realized experimentally in magnetic materials [23-26]. Their transport properties have been measured, including their electric Hall conductivity [27-32], thermal Hall conductivity, and angular momentum $[33,34]$. The identification of these quantities is often subtle, due to the Skyrmions' extended nature and their interactions with conduction electrons and other backgrounds, and has been based on phenomenological models rather than first principles. A better theoretical understanding of the transport properties of Skyrmions and their various relationships could be of great help in interpreting these experiments.

We will derive from the advertised Ward identities a set of simple and universal relations among the observables mentioned above, in the presence of baby Skyrmions (Skyrmions in $2+1$ dimensions), which we hereafter refer to simply as Skyrmions. In particular, we will argue that in insulators with translation and rotation invariance, the thermal Hall conductivity is proportional to the topological charge density, as in Eq. (8). In the absence of translation invariance, these two quantities appear in a relation (11) which also involves the Hall viscosity and angular momentum. According to our Ward identity, the Hall viscosity, which has been previously overlooked in both theoretical and experimental studies of Skyrmion systems, can be directly determined from the Skyrmion density and the thermal Hall conductivity measured as a function of momentum, as explained in Eq. (12).

For metallic materials with Skyrmions, the tight binding between Skyrmion spins and the spins of conduction electrons implies that the Skyrmion density makes a contribution to the electric Hall conductivity [27-32]. We identify this contribution in our context in Eq. (18), along with new experimental implications that should be possible to verify without difficulty. An expression for the Hall viscosity in terms of the measured electric Hall conductivity as a function of momentum is given in (21). Skyrmion dynamics turns out to be robust against the presence of impurities [35]; thus, our conclusions should apply to realistic materials.

Ward identities and central extension.-Topologically nontrivial objects can lead to significant modifications in certain physical quantities. Baby Skyrmions carry topological charge that modifies the commutators of momentum operators $[12,36,37]$

$$
\left[P^{i}\left(x^{0}\right), P^{j}\left(x^{0}\right)\right]=i \hbar C^{i j}
$$

where $x^{0}$ is time, $x^{\mu}=\left(x^{0}, \vec{x}\right)$, and $i, j=1,2$. $P^{i}\left(x^{0}\right) \equiv$ $\int d^{2} x T^{0 i}\left(x^{\mu}\right)$ is the momentum two-vector, defined as the space integral of the momentum density components of the stress energy tensor $T^{\mu \nu}$. The central term on the right side 
of (1) is proportional to the net topological charge $C^{i j}$, which may be written as the spatial integral of the topological charge density $C^{i j}=\int d^{2} x c^{i j}\left(x^{\mu}\right)$. In terms of the spin configuration $\vec{n}\left(x^{\mu}\right)$,

$$
c^{i j}=\vec{n} \cdot\left[\partial_{i} \vec{n} \times \partial_{j} \vec{n}\right] .
$$

We assume that the spin $\vec{n}$ varies smoothly over space and that the continuum description is valid on length scales much larger than the lattice spacing [26]. Since $c^{i j}$ is antisymmetric, we can write $c^{i j} \equiv c \epsilon^{i j}$. The modified commutator (1) implies that one cannot fully specify the momentum of the object due to uncertainty relations among different components. It is strongly reminiscent of the momentum algebra in the presence of a background magnetic field. As we will explain below, $c^{i j}$ can be interpreted as an effective magnetic field produced by the Skyrmions.

A local form of the momentum commutator (1) is more convenient for our purposes. As proposed in Ref. [12],

$$
\begin{aligned}
& {\left[T^{0 i}\left(x^{0}, \vec{x}\right), T^{0 j}\left(x^{0}, \vec{x}^{\prime}\right)\right]} \\
& \quad=i\left[-\partial_{i} T^{0 j}\left(x^{\mu}\right)+\partial_{j} T^{0 i}\left(x^{\mu}\right)+c^{i j}\right] \delta^{2}\left(\vec{x}-\vec{x}^{\prime}\right),
\end{aligned}
$$

where the momentum density operators $T^{0 i}\left(x^{\mu}\right)$ produce the derivative terms on the right side. Here and henceforth, we set $\hbar=1$.

We now proceed to generalize the Ward identities for systems with broken parity [5-11] by including the central extension in the equal time commutator. For simplicity, time and space translation symmetries are assumed along with rotational symmetry in the spatial plane, which is compatible with the central extension in Eq. (3) in $2+1$ dimensions [12]. We begin by considering the retarded correlator of momentum densities

$$
G^{0 i, 0 j}\left(x^{\mu}, x^{\prime \mu}\right) \equiv i \theta\left(x^{0}-x^{\prime 0}\right)\left\langle\left[T^{0 i}\left(x^{\mu}\right), T^{0 j}\left(x^{\prime \mu}\right)\right]\right\rangle .
$$

Applying two time derivatives $\partial_{0} \partial_{0}^{\prime}$ to this expression produces four terms. When both derivatives act on the momentum density, we use local momentum conservation $\partial_{\mu} T^{\mu i}=0$ [38] to obtain a term with two spatial derivatives of the retarded correlator $\partial_{n} \partial_{m}^{\prime} G^{n i, m j}$. All other terms contain a delta function $\delta\left(x^{0}-x^{\prime 0}\right)$, the derivative of the step function in (4). These latter terms are precisely the contact terms that have been reported to be missing in some evaluations of Kubo formulas [8]. We stress that our Ward identities automatically produce all possible contact terms. For translation invariant systems, all the contact terms vanish [11] and the Ward identity becomes, in momentum space,

$$
\omega^{2} \tilde{G}^{0 i, 0 j}=-i \omega \epsilon^{i j} c+q_{m} q_{n} \tilde{G}^{n i, m j},
$$

where $i, j, m, n$ are spatial indices and a Fourier transform has been applied in the form $\tilde{G}^{0 i, 0 j}\left(q_{\mu}\right) \equiv$ $\int d x^{0} d^{2} x e^{i q_{\mu} x^{\mu}} G^{0 i, 0 j}\left(x^{\mu}\right)$, with $q_{0} \equiv \omega$. We may treat the
Skyrmion density $c$ as a constant when discussing transport measurements on distance scales much larger than that of an individual Skyrmion.

Rotation invariance constrains the components of the retarded Green's function. In general, $\tilde{G}^{n i, m j}$ can have three independent contributions: a shear viscosity term $-i \omega \eta\left(\delta^{n m} \delta^{i j}+\delta^{n j} \delta^{i m}-\delta^{n i} \delta^{m j}\right)$, a bulk viscosity term $-i \omega \zeta \delta^{n i} \delta^{m j}$, and a Hall viscosity term $-(i / 2) \omega \eta_{H}\left(\epsilon^{n m} \delta^{i j}+\right.$ $\left.\epsilon^{n j} \delta^{i m}+\epsilon^{i m} \delta^{n j}+\epsilon^{i j} \delta^{n m}\right)$ [39]. The coefficients $\eta$, $\zeta$, and $\eta_{H}$ can be complex functions of the frequency, whose real parts are the usual transport coefficients. The components with two spatial indices $\tilde{G}^{0 i, 0 j}$ take the form

$-i \omega\left[\delta^{i j} \boldsymbol{\kappa}_{\delta}+\epsilon^{i j} \boldsymbol{\kappa}_{\epsilon}+q^{i} q^{j} \boldsymbol{\kappa}_{q}+\left(q^{i} \epsilon^{j n}+q^{j} \epsilon^{i n}\right) q_{n} \boldsymbol{\kappa}_{q \epsilon}\right]$,

where the form factors $\boldsymbol{\kappa}$ are the (complex) thermal conductivities, including the Hall component $\boldsymbol{\kappa}_{\epsilon}$. These form factors are analogous to the electric conductivities coming from a retarded current-current correlator that will be introduced later. By using these expressions to decompose Eq. (5) into independent tensor structures, one obtains

$$
\begin{aligned}
& \omega^{2}\left[\delta^{j l} \boldsymbol{\kappa}_{\delta}+\epsilon^{j l} \boldsymbol{\kappa}_{\epsilon}+q^{j} q^{l} \boldsymbol{\kappa}_{q}+\left(\epsilon^{j o} q^{l}+\epsilon^{l o} q^{j}\right) q_{o} \boldsymbol{\kappa}_{q \epsilon}\right] \\
& \quad=\epsilon^{j l}\left[c+q^{2} \eta_{H}\right]+\delta^{j l} q^{2} \eta+q^{j} q^{l} \zeta,
\end{aligned}
$$

which contains four distinct Ward identities corresponding to four independent tensor structures [11].

By isolating the momentum-independent terms in (7) proportional to $\delta^{j l}$ and $\epsilon^{j l}$, we arrive at the simple relations

$$
\omega^{2} \boldsymbol{\kappa}_{\delta}^{(0)}=0, \quad \omega^{2} \boldsymbol{\kappa}_{\epsilon}^{(0)}=c,
$$

where the superscript (0) denotes the momentumindependent part. Intuitively, the reason $\boldsymbol{\kappa}_{\delta}^{(0)}$ vanishes and $\boldsymbol{\kappa}_{\epsilon}^{(0)}$ does not is that Skyrmions are associated with spontaneously broken translation symmetry along with broken parity, whose imprints can only enter through the parity odd part of the conductivity at zero momentum. More precisely, the second identity predicts that the formation of a single Skyrmion results in the creation of a unit of thermal Hall conductivity $\boldsymbol{\kappa}_{\epsilon}^{(0)}$ in units of the quantized topological charge density. The frequency dependence is a consequence of the pole structure of the Goldstone boson that manifests itself in the retarded momentum correlator. In the presence of disorder, the behavior $\boldsymbol{\kappa}_{\epsilon}^{(0)}=c / \omega^{2}$ could, in principle, be lifted. However, recent numerical simulations have confirmed that Skyrmion motions are unaffected by impurities, in contrast to the case of domain walls [35]. The thermal Hall conductivity $\boldsymbol{\kappa}_{\epsilon}$ is dissipationless and exists even at zero temperature. While our Ward identity relations are valid at finite temperatures as well, measurements will be cleaner at very low temperatures, where additional dissipative contributions are suppressed. Another interpretation of Eq. (8) is that the Skyrmions carrying the thermal current propagate in 
an effective magnetic field given by the Skyrmion charge density $c_{i j}$, leading to a thermal Hall effect [40].

For the momentum-dependent terms in (7), we obtain

$$
\omega^{2} \overline{\boldsymbol{\kappa}}_{\delta}=q^{2} \eta, \quad \omega^{2} \overline{\boldsymbol{\kappa}}_{\epsilon}=q^{2} \eta_{H}, \quad \omega^{2} \boldsymbol{\kappa}_{q}=\zeta,
$$

where the overbar indicates the nonconstant momentumdependent part; for example, $\overline{\boldsymbol{\kappa}}_{\epsilon}=\boldsymbol{\kappa}_{\epsilon}-\boldsymbol{\kappa}_{\epsilon}^{(0)}=q^{2} \boldsymbol{\kappa}_{\epsilon}^{(2)}+$ $q^{4} \boldsymbol{\kappa}_{\epsilon}^{(4)}+\cdots$. Thus, thermal conductivities are directly connected to the viscosities of the system, as previously confirmed [11]. Furthermore, it follows from (7) that $\boldsymbol{\kappa}_{q \epsilon}=0$.

If the system of interest is not translationally invariant, there will be additional contributions to the Ward identity (9); however, the zero momentum identity (8) will be unmodified. A particularly interesting contribution of this type arises in parity-breaking systems exhibiting spontaneously generated angular momentum $\ell[36,41]$, where the momentum generator can develop an expectation value

$$
\left\langle T^{0 i}\right\rangle=\frac{1}{2} \epsilon^{i k} \partial_{k} \ell .
$$

In the absence of translation invariance, the two time derivatives $\partial_{0} \partial_{0}^{\prime}$ acting on $G^{0 i, 0 j}\left(x^{\mu}, x^{\prime \mu}\right)$ pick up the contact term $i / 2\left(\partial_{0}^{\prime}-\partial_{0}\right)\left[\delta\left(x^{0}-x^{\prime 0}\right)\left\langle\left[T^{0 i}\left(x^{\mu}\right), T^{0 j}\left(x^{\prime \mu}\right)\right]\right\rangle\right]$ in addition to the terms appearing in (5). The commutator yields a tensor similar to $\eta_{H}$ coming from the last term in (5), as one can check using (3) and (10) [11]. As a result, $\eta_{H}$ in (9) is modified to $\eta_{H}+\ell / 2$. In such cases, a coordinate space description might be more convenient. Similarly, the inclusion of pressure $p$, another universal contribution, would replace $\zeta$ in the last relation of (9) by the combination $\zeta-(i / \omega) p$.

Ward identities for insulators.-Recently, Skyrmions have been observed in the insulating material $\mathrm{Cu}_{2} \mathrm{OSeO}_{3}$ [42], and various experiments regarding the Hall thermal conductivity and angular momentum have been carried out $[34,43,44]$. For insulators, our Ward identity provides a simple relation among parity violating transport coefficients

$$
\omega^{2} \boldsymbol{\kappa}_{\epsilon}=c-\partial^{2}\left(\eta_{H}+\frac{\ell}{2}\right)
$$

which is derived from Eqs. (7) and (10) in the absence of translation invariance. Recent experiments have successfully measured the Skyrmion density, thermal Hall conductivity, and angular momentum in Skyrmion materials [34]. Such measurements could, in principle, be used to infer the existence of Hall viscosity [45].

In the presence of translation invariance, there is a simple way to measure the Hall viscosity. Combining Eqs. (8) and (9), we get

$$
\eta_{H}=c \frac{\overline{\boldsymbol{\kappa}}_{\epsilon}}{q^{2} \boldsymbol{\kappa}_{\epsilon}^{(0)}} \rightarrow c \frac{\boldsymbol{\kappa}_{\epsilon}^{(2)}}{\boldsymbol{\kappa}_{\epsilon}^{(0)}},
$$

where we take the limit $q^{2} \rightarrow 0$. Once the thermal Hall conductivity $\boldsymbol{\kappa}_{\epsilon}$ is measured as a function of $q^{2}$, the Hall viscosity is nothing but the Skyrmion density multiplied by the ratio between the slope and the $\boldsymbol{\kappa}_{\epsilon}$-intercept $\boldsymbol{\kappa}_{\epsilon}\left(q^{2}=0\right)$. Note that this is only applicable in the presence of nonzero Skyrmion density.

Ward identities for conductors.-The neutral case discussed above provides a simple relation between the topological charge density and thermal Hall conductivity. However, since most realistic materials reveal Skyrmions in the presence of electric charge carriers, we need to generalize our discussion to include conducting materials. We will see that Skyrmions have a direct effect on charged dynamics as well, which can be accounted for by the inclusion of a conserved $U(1)$ current $J^{\mu}, \partial_{\mu} J^{\mu}=0$.

In the presence of a uniform external magnetic field $B$, the momentum density gets modified to

$$
T_{B}^{0 j}=T^{0 j}-(B / 2) \epsilon_{k}^{j} x^{k} J^{0} .
$$

This modification is the expected minimal coupling in the presence of a constant magnetic field. Another important modification appears in the conservation equation

$$
\partial_{\mu} T^{\mu i}=B \epsilon_{j}^{i} J^{j} .
$$

These are the spatial components of the general relation $\partial_{\mu} T^{\mu \nu}=F^{\nu \rho} J_{\rho}$. The Ward identities are once again obtained by taking time derivatives of the correlator (4). The derivation is straightforward, and we present the details in the Supplemental Material [47]. The resulting full Ward identity is

$$
\begin{aligned}
\delta^{j l} & {\left[\omega^{2} \boldsymbol{\kappa}_{\delta}+i \omega B\left(\boldsymbol{\alpha}_{\epsilon}+\boldsymbol{\alpha}_{\epsilon}^{*}+q^{2}\left[\boldsymbol{\alpha}_{q \epsilon}-\boldsymbol{\alpha}_{q \epsilon}^{*}\right]\right)+B^{2}\left(\boldsymbol{\sigma}_{\delta}+q^{2} \boldsymbol{\sigma}_{q}\right)\right] } \\
& +\epsilon^{j l}\left[\omega^{2} \boldsymbol{\kappa}_{\epsilon}-i \omega B\left(\boldsymbol{\alpha}_{\delta}+\boldsymbol{\alpha}_{\delta}^{*}+q^{2}\left[\boldsymbol{\alpha}_{q}+\boldsymbol{\alpha}_{q}^{*}\right] / 2\right)+B^{2} \boldsymbol{\sigma}_{\epsilon}\right] \\
& +q^{j} q^{l}\left[\omega^{2} \boldsymbol{\kappa}_{q}-2 i \omega B\left(\boldsymbol{\alpha}_{q \epsilon}-\boldsymbol{\alpha}_{q \epsilon}^{*}\right)-B^{2} \boldsymbol{\sigma}_{q}\right] \\
& +\left(\epsilon^{j o} q^{l}+\epsilon^{l o} q^{j}\right) q_{o}\left[\omega^{2} \boldsymbol{\kappa}_{q \epsilon}+i \omega B\left(\boldsymbol{\alpha}_{q}-\boldsymbol{\alpha}_{q}^{*}\right) / 2-B^{2} \boldsymbol{\sigma}_{q \epsilon}\right] \\
= & \epsilon^{j l}\left[c-B \rho+q^{2} \eta_{H}\right]+\delta^{j l} q^{2} \eta+q^{j} q^{l} \zeta,
\end{aligned}
$$

where $\boldsymbol{\alpha}, \boldsymbol{\alpha}^{*}$ are thermoelectric conductivity tensors related to the form factors of momentum-current correlators $G^{0 i, j} \sim\left\langle\left[T^{0 i}, J^{j}\right]\right\rangle$ and $G^{i, 0 j} \sim\left\langle\left[J^{i}, T^{0 j}\right]\right\rangle$, while the $\boldsymbol{\sigma}^{\text {'s are }}$ electric conductivity tensors associated with current-current correlators $G^{i, j} \sim\left\langle\left[J^{i}, J^{j}\right]\right\rangle$. They arise due to the modifications in equations (13) and (14) and the corresponding mix between the momentum $T^{0 i}$ and charge $J^{j}$ densities. There are four independent tensor structures and four corresponding Ward identities in (15). These identities reduce to those of insulators when $B=0$, Eq. (7). 
The momentum-independent Ward identities give

$$
\begin{aligned}
& \omega^{2} \boldsymbol{\kappa}_{\delta}^{(0)}+i \omega B\left(\boldsymbol{\alpha}_{\epsilon}^{(0)}+\boldsymbol{\alpha}_{\epsilon}^{*(0)}\right)+B^{2} \boldsymbol{\sigma}_{\delta}^{(0)}=0, \\
& \omega^{2} \boldsymbol{\kappa}_{\epsilon}^{(0)}-i \omega B\left(\boldsymbol{\alpha}_{\delta}^{(0)}+\boldsymbol{\alpha}_{\delta}^{*(0)}\right)+B^{2} \boldsymbol{\sigma}_{\epsilon}^{(0)}=c-B \rho,
\end{aligned}
$$

which reduce to Eq. (8) when $B=0$. At nonzero momentum, there are four independent relations connecting viscosities and conductivities as in the neutral case. In particular, the Hall viscosity [39] is

$q^{2} \eta_{H}=\omega^{2} \overline{\boldsymbol{\kappa}}_{\epsilon}+B^{2} \overline{\boldsymbol{\sigma}}_{\epsilon}-i \omega B\left(\overline{\boldsymbol{\alpha}}_{\delta}+\overline{\boldsymbol{\alpha}}_{\delta}^{*}+\frac{\boldsymbol{\alpha}_{q}+\boldsymbol{\alpha}_{q}^{*}}{2}\right)$.

Ward identities for conductors at zero momentum.-In Refs. [26,48,49], interactions between Skyrmions and conduction electrons are modeled by the ferromagnetic spin coupling. In the strong coupling limit, the spin wave function of the conduction electrons is identified with that of the localized spin $\vec{n}\left(x^{\mu}\right)$ of the Skyrmions. This limit is described by a tight binding model with Hund's rule coupling. More general interactions between conduction electrons and local magnetization may be considered [50].

We will discuss two different ways to model the effects of the interaction between the thermal and charge responses. First, we can modify the parameters of the Ward identities. The Skyrmion charge density produces an emergent magnetic field $b=c / 2$ [26], which can change the dynamics of conduction electrons, similarly to $B$. For simplicity, we assume that the emergent magnetic field is homogeneous and constant, which is the case for all practical measurements. Because of the tight binding, the motion of the conduction electrons will also influence the thermal response of the Skyrmions. At vanishing momentum, by taking these effects into account, we get

$\omega^{2} \boldsymbol{\kappa}_{\epsilon}^{(0)}-i \omega B_{b}\left(\boldsymbol{\alpha}_{\delta}^{(0)}+\boldsymbol{\alpha}_{\delta}^{*(0)}\right)+B_{b}^{2} \boldsymbol{\sigma}_{\epsilon}^{(0)}=c_{b}-B_{b} \rho$.

This identity is of the same form as (16), with the modification $B \rightarrow B_{b} \equiv B+b$ contributing to the charge response and $c \rightarrow c_{b} \equiv c+c_{\mathrm{el}}$ incorporating an additional contribution to the thermal response from the conduction electrons $c_{\mathrm{el}}$, without changing the topological charge density. The quantities $c$ and $b$ are constant and independent of $B$, while $c_{\text {el }}$ (also measurable) is expected to be proportional to $B$ and depends on the strength of the binding. $b, c, c_{\mathrm{el}}$ are expected to be readily identifiable experimentally. In particular, $b$ can be identified from a step-function-like signature in the Hall conductivity $\boldsymbol{\sigma}_{\epsilon}$ [27-30], as one passes into and out of a phase in which Skyrmions develop a finite density $c$. Such behavior will also confirm the presence of a nonzero density $c$, which will likewise produce a similar step-function-like contribution in the thermal Hall conductivity $\boldsymbol{\kappa}_{\epsilon}$ with an additional
$B$-dependent $c_{\mathrm{el}}$, by sweeping the magnetic field $B$ or the temperature $T$ independently.

In the absence of ferromagnetic binding between the Skyrmion and conduction electron spins, the electric Hall conductivity would only pick up contributions from the conduction electrons, and $B_{b}$ would reduce to $B$. On the other hand, the thermal Hall conductivity would include both contributions $c$ and $c_{\mathrm{el}}$, with the latter being independent of $B$.

A second, alternative way to incorporate the interaction between thermal and charged responses is to impose the following operator relation

$$
T^{0 i}=\mu J^{i},
$$

where $\mu$ parametrizes the strength of the coupling between the spins of the Skyrmion and the conduction electron. Then, the momentum transport is tied to the charge transport as $\boldsymbol{\kappa}=\mu \alpha=\mu \alpha^{*}=\mu^{2} \boldsymbol{\sigma}$ [51]. The relation (19) implies a distinct experimental signature. At zero momentum, the analogue of (18) becomes

$$
\boldsymbol{\sigma}_{\delta}^{(0)}=-\frac{i \omega_{c}}{\omega} \frac{c-B \rho}{\mu^{2}\left(\omega^{2}-\omega_{c}^{2}\right)}, \quad \boldsymbol{\sigma}_{\epsilon}^{(0)}=\frac{c-B \rho}{\mu^{2}\left(\omega^{2}-\omega_{c}^{2}\right)},
$$

where $\omega_{c}=B / \mu$. For small magnetic field $\omega_{c} \ll \omega$, the Hall conductivity is directly related to the topological charge density $\boldsymbol{\sigma}_{\epsilon}^{(0)} \approx c / \mu^{2} \omega^{2}$ and $\boldsymbol{\sigma}_{\delta}^{(0)} \approx 0$. In the opposite limit with large magnetic field $\omega_{c} \gg \omega, \boldsymbol{\sigma}_{\epsilon}^{(0)} \approx 0$ and $\boldsymbol{\sigma}_{\delta}^{(0)} \approx-i \rho / \mu \omega$. Such behavior can easily be measured. It would be interesting to find a material with Skyrmions that displays these properties.

The momentum-independent Ward identities are the same as (16) whether or not the system has translation invariance. If the system of interest has translation symmetry, one can use (17) with the modification $B \rightarrow B_{b}$ for momentum-dependent Hall transport measurements.

In the presence of translation symmetry, it is also simple to measure the Hall viscosity similarly to Eq. (12). Dividing Eq. (17) by the second equation of (16) with $B \rightarrow B_{b}$, $c \rightarrow c_{b}$, and taking the approximation $\omega / B_{b} \rightarrow 0$ and the limit $q^{2} \rightarrow 0$, we obtain

$$
\eta_{H}=\left(c_{b}-B_{b} \rho\right) \frac{\boldsymbol{\sigma}_{\epsilon}^{(2)}}{\boldsymbol{\sigma}_{\epsilon}^{(0)}}
$$

In the opposite limit $B_{b} / \omega \rightarrow 0, \eta_{H}$ reduces to Eq. (12) with the modification $c \rightarrow c_{b}-B_{b} \rho$. Note that this identification of $\eta_{H}$ can also be applied to systems without Skyrmions, such as quantum Hall systems.

Ward identities for conductors without translation invariance.-If spatial translation symmetry is broken (still assuming time translation and rotation invariance), more physical quantities can come into play. In particular, the 
Ward identity is given by Eq. (17) with the replacement $\eta_{H} \rightarrow \eta_{H}+\ell / 2$ as in the neutral case [52]. This Ward identity directly relates conductivities, angular momentum, and Hall viscosity. Recent experiments on metallic $\mathrm{MnSi}$ have studied transport properties and angular momentum $[33,53]$. While Hall viscosity has not previously been discussed in the context of Skyrmion physics, it might play an important role and has a chance to be observed for the first time in active experiments.

We thank Sumit Das, Lance De Long, Michael Eides, Ori Ganor, Petr Hořava, Carlos Hoyos, Seungjoon Hyun, Seok Kim, Elias Kiritsis, Kimyeong Lee, Keh-Fei Liu, Hitoshi Murayama, Yaron Oz, Sumiran Pujari, Shinsei Ryu, Sang-Jin Sin, David Tong, Oskar Vafek, and Piljin Yi for helpful discussions. We are especially thankful to Jung Hoon Han for numerous illuminating discussions on Skyrmions and invaluable comments on the draft. Finally, we would like to thank our referees for numerous helpful comments. B. S. K. is grateful to the members of the Berkeley Center for Theoretical Physics, Berkeley, and KIAS, Seoul, for their warm hospitality during his visits. This work is partially supported by NSF Grant No. PHY1214341.

[1] J. C. Ward, An identity in quantum electrodynamics, Phys. Rev. 78, 182 (1950).

[2] Y. Takahashi, On the generalized Ward identity, Nuovo Cimento 6, 371 (1957).

[3] Y. Nambu, Quasi-particles and gauge invariance in the theory of superconductivity, Phys. Rev. 117, 648 (1960).

[4] H. K. Janssen, B. Schaub, and B. Schmittmann, New universal short-time scaling behaviour of critical relaxation processes, Z. Phys. B 73, 539 (1989).

[5] N. Read, Non-Abelian adiabatic statistics and Hall viscosity in quantum Hall states and $p(x)+i p(y)$ paired superfluids, Phys. Rev. B 79, 045308 (2009).

[6] N. Read and E. H. Rezayi, Hall viscosity, orbital spin, and geometry: Paired superfluids and quantum Hall systems, Phys. Rev. B 84, 085316 (2011).

[7] C. Hoyos and D. T. Son, Hall Viscosity and Electromagnetic Response, Phys. Rev. Lett. 108, 066805 (2012).

[8] B. Bradlyn, M. Goldstein, and N. Read, Kubo formulas for viscosity: Hall viscosity, Ward identities, and the relation with conductivity, Phys. Rev. B 86, 245309 (2012).

[9] M. Geracie, D. T. Son, C. Wu, and S. F. Wu, Spacetime symmetries of the quantum Hall effect, Phys. Rev. D 91, 045030 (2015).

[10] C. Hoyos, B. S. Kim, and Y. Oz, Ward identities for Hall transport, J. High Energy Phys. 10 (2014) 054.

[11] C. Hoyos, B.S. Kim, and Y. Oz, Ward identities for transport in $2+1$ dimensions, J. High Energy Phys. 03 (2015) 164.

[12] H. Watanabe and H. Murayama, Noncommuting Momenta of Topological Solitons, Phys. Rev. Lett. 112, 191804 (2014).
[13] T. H. R. Skyrme, A non-linear theory of strong interactions, Proc. R. Soc. A 247, 260 (1958).

[14] A. A. Belavin and A. M. Polyakov, Metastable states of twodimensional isotropic ferromagnets, JETP Lett. 22, 245 (1975).

[15] F. Wilczek and A. Zee, Linking Numbers, Spin, and Statistics of Solitons, Phys. Rev. Lett. 51, 2250 (1983).

[16] A. Bogdanov and D. Yablonskii, Thermodynamically stable "vortices", in magnetically ordered crystals: The mixed state of magnets, JETP 68, 101 (1989).

[17] A. N. Bogdanov, U. K. Rössler, M. Wolf, and K.-H. Müller, Magnetic structures and reorientation transitions in noncentrosymmetric uniaxial antiferromagnets, Phys. Rev. B 66, 214410 (2002).

[18] U. Rössler, A. Bogdanov, and C. Pfleiderer, Spontaneous Skyrmion ground states in magnetic metals, Nature (London) 442, 797 (2006).

[19] B. Binz, A. Vishwanath, and V. Aji, Theory of the Helical Spin Crystal: A Candidate for the Partially Ordered State of MnSi, Phys. Rev. Lett. 96, 207202 (2006).

[20] B. Binz and A. Vishwanath, Theory of helical spin crystals: Phases, textures, and properties, Phys. Rev. B 74, 214408 (2006).

[21] S. Tewari, D. Belitz, and T. R. Kirkpatrick, Blue Quantum Fog: Chiral Condensation in Quantum Helimagnets, Phys. Rev. Lett. 96, 047207 (2006).

[22] I. Fischer, N. Shah, and A. Rosch, Crystalline phases in chiral ferromagnets: Destabilization of helical order, Phys. Rev. B 77, 024415 (2008).

[23] S. Mühlbauer, B. Binz, F. Jonietz, C. Pfleiderer, A. Rosch, A. Neubauer, R. Georgii, and P. Böni, Skyrmion lattice in a chiral magnet, Science 323, 915 (2009).

[24] X. Z. Yu, Y. Onose, N. Kanazawa, J. H. Park, J. H. Han, Y. Matsui, N. Nagaosa, and Y. Tokura, Real-space observation of a two-dimensional Skyrmion crystal, Nature (London) 465, 901 (2010).

[25] S. Heinze, K. von Bergmann, M. Menzel, J. Brede, A. Kubetzka, R. Wiesendanger, G. Bihlmayer, and S. Blügel, Spontaneous atomic-scale magnetic Skyrmion lattice in two dimensions, Nat. Phys. 7, 713 (2011).

[26] N. Nagaosa and Y. Tokura, Topological properties and dynamics of magnetic Skyrmions, Nat. Nanotechnol. 8, 899 (2013).

[27] M. Lee, W. Kang, Y. Onose, Y. Tokura, and N. P. Ong, Unusual Hall anomaly in MnSi under pressure, Phys. Rev. Lett. 102, 186601 (2009).

[28] A. Neubauer, C. Pfleiderer, B. Binz, A. Rosch, R. Ritz, P. G. Niklowitz, and P. Böni, Topological Hall Effect in the A Phase of MnSi, Phys. Rev. Lett. 102, 186602 (2009).

[29] N. Kanazawa, Y. Onose, T. Arima, D. Okuyama, K. Ohoyama, S. Wakimoto, K. Kakurai, S. Ishiwata, and Y. Tokura, Large Topological Hall Effect in a Short-Period Helimagnet MnGe, Phys. Rev. Lett. 106, 156603 (2011).

[30] T. Schulz, R. Ritz, A. Bauer, M. Halder, M. Wagner, C. Franz, C. Pfleiderer, K. Everschor, M. Garst, and A. Rosch, Emergent electrodynamics of Skyrmions in a chiral magnet, Nat. Phys. 8, 301 (2012).

[31] Y. Li, N. Kanazawa, X. Z. Yu, A. Tsukazaki, M. Kawasaki, M. Ichikawa, X. F. Jin, F. Kagawa, and Y. Tokura, Robust Formation of Skyrmions and Topological Hall Effect 
Anomaly in Epitaxial Thin Films of MnSi, Phys. Rev. Lett. 110, 117202 (2013).

[32] S. X. Huang and C. L. Chien, Extended Skyrmion Phase in Epitaxial FeGe(111) Thin Films, Phys. Rev. Lett. 108, 267201 (2012).

[33] F. Jonietz, S. Mühlbauer, C. Pfleiderer, A. Neubauer, W. Mnzer, A. Bauer, T. Adams, R. Georgii, P. Böni, R. A. Duine, K. Everschor, M. Garst, and A. Rosch, Spin transfer torques in $\mathrm{MnSi}$ at ultralow current densities, Science 330, 1648 (2010).

[34] M. Mochizuki, X.Z. Yu, S. Seki, N. Kanazawa, W. Koshibae, J. Zang, M. Mostovoy, Y. Tokura, and N. Nagaosa, Thermally driven ratchet motion of a Skyrmion microcrystal and topological magnon Hall effect, Nat. Mater. 13, 241 (2014).

[35] J. Iwasaki, M. Mochizuki, and N. Nagaosa, Universal current-velocity relation of Skyrmion motion in chiral magnets, Nat. Commun. 4, 1463 (2013).

[36] N. Papanicolaou and T. N. Tomaras, Dynamics of magnetic vortices, Nucl. Phys. B360, 425 (1991).

[37] D. J. Thouless, P. Ao, and Q. Niu, Transverse Force on a Quantized Vortex in a Superfluid, Phys. Rev. Lett. 76, 3758 (1996).

[38] Although translation symmetry is spontaneously broken due to the presence of Skyrmions, the conservation equations can be checked to hold with the model in Ref. [12]. Translation symmetry of the Skyrmion configuration can be restored in the continuum limit.

[39] J. E. Avron, R. Seiler, and P. G. Zograf, Viscosity of Quantum Hall Fluids, Phys. Rev. Lett. 75, 697 (1995).

[40] M. Stone, Magnus force on Skyrmions in ferromagnets and quantum Hall systems, Phys. Rev. B 53, 16573 (1996).

[41] H. Liu, H. Ooguri, B. Stoica, and N. Yunes, Spontaneous Generation of Angular Momentum in Holographic Theories, Phys. Rev. Lett. 110, 211601 (2013).

[42] S. Seki, X. Z. Yu, S. Ishiwata, and Y. Tokura, Observation of Skyrmions in a multiferroic material, Science 336, 198 (2012).

[43] S. Seki, J.-H. Kim, D. S. Inosov, R. Georgii, B. Keimer, S. Ishiwata, and Y. Tokura, Formation and rotation of
Skyrmion crystal in the chiral-lattice insulator $\mathrm{Cu}_{2} \mathrm{OSeO}_{3}$, Phys. Rev. B 85, 220406(R) (2012).

[44] K. A. van Hoogdalem, Y. Tserkovnyak, and D. Loss, Magnetic texture-induced thermal Hall effects, Phys. Rev. B 87, 024402 (2013).

[45] In real materials, $\boldsymbol{\kappa}_{\epsilon}$ has a further contribution from the magnon [42], whose dynamics has been considered recently [46]. Modeling the interaction between the Skyrmion and the magnon in this context of Ward identity is an interesting problem, which is beyond the scope of this Letter due to the nonconservation of the magnon spin and the dependence on details of the interaction.

[46] Y.-T. Oh, H. Lee, J.-H. Park, and J. H. Han, Dynamics of magnon fluid in Dzyaloshinskii-Moriya magnet and its manifestation in magnon-Skyrmion scattering, Phys. Rev. B 91, 104435 (2015).

[47] See Supplemental Material at http://link.aps.org/ supplemental/10.1103/PhysRevLett.117.116805 for the derivation of the full Ward identity in the presence of a magnetic field and current.

[48] G. E. Volovik, Linear momentum in ferromagnets, J. Phys. C 20, L83 (1987).

[49] J. Zang, M. Mostovoy, J. H. Han, and N. Nagaosa, Dynamics of Skyrmion Crystals in Metallic Thin Films, Phys. Rev. Lett. 107, 136804 (2011).

[50] S. Zhang and Z. Li, Role of Nonequilibrium Conduction Electrons on the Magnetization Dynamics of Ferromagnets, Phys. Rev. Lett. 93, 127204 (2004).

[51] Similar relations to (19) have been used to impose Galilean invariance in related systems [5-11].

[52] Likewise, the magnetization $M$, from the expectation value of the current $\left\langle J^{i}\right\rangle=\epsilon^{i k} \partial_{k} M$, can contribute in the combination $\zeta-(i / \omega)(p-B M)$, replacing the bulk viscosity $\zeta$ [11].

[53] K. Everschor, M. Garst, B. Binz, F. Jonietz, S. Mühlbauer, C. Pfleiderer, and A. Rosch, Rotating Skyrmion lattices by spin torques and field or temperature gradients, Phys. Rev. B 86, 054432 (2012). 